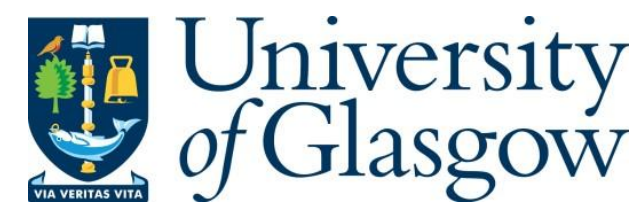

Zare-Behtash, H., Yang, L., Gongora-Orozco, N., Kontis, K., and Jones, A. (2012) Anodized aluminium pressure sensitive paint: effect of paint application technique. Measurement, 45 (7). pp. 1902-1905. ISSN 0263 2241(doi:10.1016/j.measurement.2012.03.015)

Copyright ( 2012 Elsevier

A copy can be downloaded for personal non-commercial research or study, without prior permission or charge

Content must not be changed in any way or reproduced in any format or medium without the formal permission of the copyright holder(s)

When referring to this work, full bibliographic details must be given

http://eprints.gla.ac.uk/84622/

Deposited on: 07 January 2014

Enlighten - Research publications by members of the University of Glasgow http://eprints.gla.ac.uk 


\title{
Anodized Aluminium Pressure Sensitive Paint: Effect of Paint Application Technique
}

\author{
H. Zare-Behtash, ${ }^{*}$ L. Yang, N. Gongora-Orozco, K. Kontis, and A. Jones \\ School of Mechanical, Aerospace and Civil Engineering, University of Manchester, UK
}

\begin{abstract}
The porous surface of the Anodized Aluminium Pressure Sensitive Paint (AA-PSP) is what differentiates it from conventional sol-gel based PSPs, leading to a faster response time of the paint. The objective of the current study is to examine the effect of the paint application technique, i.e., whether the AA substrate is dipped or sprayed, on the pressure and temperature sensitivity. A more practical procedure for preparing the AA samples is also presented. Scanning Electron Microscope (SEM) images are acquired together with the calibration of the AA-PSP at various temperatures and pressures to determine the effectiveness of each application technique. The results revealed that the AA sample which was dipped in the PSP solution shows a higher pressure sensitivity than the sprayed one. The SEMs show that spraying leads to the covering up of the micropores created on the surface and undermining the benefit of anodization.
\end{abstract}

Keywords: Pressure sensitive paints, Anodized aluminium

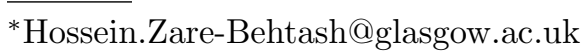




\section{INTRODUCTION}

Pressure sensitive paints offer the advantage of non-intrusive global pressure mapping of aerodynamic surfaces. ${ }^{1,2}$ Anodised Aluminium Pressure Sensitive Paint (AA-PSP) uses an electro-chemical process to develop a porous surface on the aluminium substrate to which the oxygen sensitive paint is added. ${ }^{3-7}$ The porous surface leads to an increased surface area and hence greater chance of interaction between the oxygen molecules in the surrounding environment and the oxygen sensitive luminophores molecules in the paint. ${ }^{8}$ This leads to an increased response time of the pressure sensitive paint, a crucial parameter in the analysis of high speed unsteady flows.

Traditionally the anodized model is dipped in the PSP solution where it is allowed to adsorb the oxygen sensitive molecules present in the solution. ${ }^{9}$ The process of dipping has costly limitations. If the model is relatively large, e.g., in order to study unsteadiness in detail, then large volumes of PSP must be prepared so the entire model can be dipped into the solution. Due to the expensive nature of the oxygen sensitive molecules, in this case Ruthenium, making large volumes of PSP to cover the entire model will be very expensive. Also, if one is only interested in obtaining the pressure data over a small area of interest, there is no need to prepare large volumes of PSP to dip the whole model and the area of interest can just be sprayed with PSP. Large volumes of PSP solution also raises the issue of storing the photosensitive and easily evaporating mixture. On the other hand if the model is too small then very sensitive and high resolution image acquisition apparatus is required to study the small fluctuations, again increasing costs.

The objective of the current study is two fold: firstly a more practical approach to preparing anodized aluminium samples is presented, secondly if AA-PSP samples that have been sprayed rather than dipped exhibit adequate pressure sensitivity this would lead to a reduction in PSP solution required to dip the entire model and therefore lead to reduced costs. 


\section{EXPERIMENTAL SETUP}

\section{A. Anodization Procedure}

The pre-treatment procedure of the aluminium sample to be anodized is identical to that of Sakaue ${ }^{10}$ and Kameda et al. ${ }^{11}$ In the anodization part of the procedure we have adopted a simpler approach. Where the sample is to be dipped in 1 molar sulfuric acid Kameda et al. ${ }^{11}$ recommends a constant temperature of $5-10^{\circ} \mathrm{C}$ compared to the $0^{\circ} \mathrm{C}$ used by Sakaue. ${ }^{10}$ Since maintaining a constant low temperature requires specialist equipment, we sufficed to anodizing the aluminium sample at room temperature, therefore eliminating the need for temperature regulation. The post-treatment procedure of the sample is again identical to the aforementioned researchers.

Figure 1 shows the scanning electron microscope (SEM) image of the surface profile of an aluminium sample anodized using the aforementioned procedure. The porous structure of the anodized sample is clearly evident.

\section{B. Paint Formulation}

One mol is the unit which has Avogadro's number of molecules $\left(6.02 \times 10^{23}\right)$. If we have $V(m l)$ of solvent, we need to measure $w(g)$ of dye by the following relationship:

$$
\frac{0.1}{1000}\left(\frac{m o l}{l}\right)=\left[\frac{w(g)}{F W(g / m o l)}\right] /\left[\frac{V(m l)}{1000}\right]
$$

where $F W$ is the formula weight of tris-(Bathophenanthroline) Ruthenium (II) Perchlorate $\left(\left(\mathrm{C}_{24} \mathrm{H}_{16} \mathrm{~N}_{2}\right)_{3} \mathrm{Ru}\left(\mathrm{ClO}_{4}\right)_{2}\right)$ provided by supplier (GFS Chemicals) equal to $F W=$ 1297.20. From Eq. (1), the amount of dye, w, can be determined. The solvent, $V$, was Dichloromethane or DCM $\left(\mathrm{CH}_{2} \mathrm{CL}_{2}\right)$ purchased from Acros Organics. The $0.1 \mathrm{mM}$ concentration of Ruthenium on the left hand siude of Eq. (1) is based on the work of Sakaue ${ }^{10}$ and Gongora-Orozco et al. ${ }^{12}$

Aluminium samples measuring $20 \times 20 \times 2 \mathrm{~mm}$ were used to observe the effect of the different paint application techniques. Three samples were studied: 1) dipped, 2) sprayed with 9 layers, and 3) sprayed with 30 layers. 


\section{Calibration Apparatus}

A specially designed calibration chamber was used to determine the pressure and temperature sensitivity of AA-PSP samples. The pressure was monitored with a Kulite XT-190 and varied in the range between 0.5 and 4 bar. Using a peltier heater/cooler manufactured by Greenweld, the temperature of the PSP sample was controlled was controlled in the range $264 \mathrm{~K}$ and $320 K$. A k-type thermocouple mounted on the surface of the PSP sample allowed for the continuous monitoring of the surface temperature.

A pair of light emitting diode (LED) panels with peak wavelength of 470nm were used for illuminated. Each LED panel comprised of $13 \times 10$ LEDs. The luminescent emission was captured by a CCD camera (LaVision Image Intense). The setup is identical to that used by Zare-Behtash et al. ${ }^{13}$

A combination of two filters was used to capture the emitted light. The first, an orange long pass filter, only allowing the transmission of light with $\lambda>550 \mathrm{~nm}$ and the second filter was an Infra-Red (IR) cut-off filter, preventing the transmission of light with $\lambda>700 \mathrm{~nm} .{ }^{14}$

\section{RESULTS AND DISCUSSIONS}

\section{A. Effect of Paint Application Technique}

Figure 2 presents the SEM images for two AA samples which have been coated with the PSP by the dipping and spraying methods, respectively. Both samples were originally part of the same piece of aluminium that was anodized according to the aforementioned procedure, and then cut into small square sections. The most prominent difference between the two is the surface profile; whilst the dipped sample shows a very uniform distribution the sprayed sample contains a plethora of droplets as a result of airbrushing.

Examining the intensity profiles of the various AA samples through the CCD camera we arrive at Figure 3. The dipped sample contains distinct surface non-uniformities. Due to the very thin layer of paint deposited on the sample as a result of dipping even the slightest discontinuities on the model surface are highlighted. Since in PSP analysis intensities are divided by a reference image intensity these surface non-uniformities are not difficult to deal with. However, care must be taken in instances where the PSP sample is prone to slight movements during testing. 
The sprayed samples exhibit an unexpected uniformity with both samples exhibiting a higher intensity (signal level) compared to the dipped sample. This is due to the thicker layer of PSP deposited on the sprayed samples. However, the intensity of the paint reduces when more layers are sprayed on. At high levels of luminophore concentrations adverse interactions take place among the luminophore molecules which lead to the decrease in intensity. $^{15}$

In Figure 4 using the backscatter SEM image the relatively thick layer of PSP covering the porous surface is evident, making the porous anodized surface superfluous.

\section{B. Pressure and Temperature Sensitivity}

The pressure sensitivity of PSPs is determined through a Stern-Volmer plot. The S-V plot consists of the intensity ratio of wind-off to wind-on $\left(I_{\text {ref }} / I\right)$ images plotted against pressure. The higher the gradient of each line the greater the pressure sensitivity. The S-V plot for the three AA-PSP samples tested is presented in Figure 5. The reference intensity corresponds to ambient conditions $\left(P=P_{r e f}=1 b a r\right)$. The best way to analyse this figure is by focusing on one particular temperature and observing how each sample behaves. For example at $264 K$ the dipped sample exhibits the greatest pressure sensitivity and sample with 30 layers has the lowest pressure sensitivity. This behaviour is repeated for all temperatures tested.

An ideal pressure sensitive paint would display no or little temperature sensitivity. However, most pressure sensitive paints exhibit slight temperature dependencies. Figure 6 shows the variation in intensity obtained at three different pressures for the three temperatures tested. As the results indicate the dipped AA-PSP sample exhibits the greatest variation in intensity at different temperatures whilst the model that has been sprayed with 30 layers of PSP shows the least variation in intensity with temperature.

\section{CONCLUSIONS}

Using the simpler anodization procedure presented in this paper we have been able to create a porous surface for the application of AA-PSP.

The behaviour of three AA-PSP samples which were dipped, sprayed with 9 layers, and sprayed with 30 layers were compared. SEM images of the dipped AA-PSP sample showed 
a uniform surface profile compared to the sprayed samples. The dipped sample has a relatively low signal level due to the thin layer of paint deposited on it. The dipped AA-PSP sample displays a greater pressure sensitivity and at the same time the highest temperature sensitivity. The sprayed sample with 30 layers with a high concentration of oxygen sensitive luminophore showed the lowest pressure and also temperature sensitivity.

More SEM analysis is planned to explore the three-dimensional surface texture of the anodised samples. This will give a better understanding of the dimensions of the micropores formed on the AA surface and lead to a better understanding of how best to utilise this structure to improve the efficacy of AA-PSP.

\section{Acknowledgments}

The authors are indebted to the technical staff at The University of Manchester for their assistance.

1 Bell, J.H., Schairer, E.T., Hand, L.A., Mehta, D., "Surface pressure measurements using luminescent coatings," Annual Review of Fluid Mechanics 33, pp. 155-206, 2001.

${ }^{2}$ Gregory, J.W., Asai, K., Kameda, M., Liu, T., Sullivan, J.P., "A review of pressure-sensitive paint for high-speed and unsteady aerodynamics," Proceedings of the Institute of Mechanical Engineers, Part G: Journal of Aerospace Engineering 222, pp. 249-290, 2008.

${ }^{3}$ Nakakita, K., Yamazaki, T., Asai, K., Teduka, N., Fuji, A., Kameda, M., "Pressure sensitive paint measurement in a hypersonic shock tunnel," 21st AIAA Aerodynamic Measurement Technology and Ground Testing Conference, Denvor, AIAA-2000-2523, 2000.

4 Sakaue, H., Sullivan, J.P., "Time response of anodized aluminium pressure-sensitive paint," AIAA Journal 39, pp. 1944-1949, 2001.

5 Sakaue, H., "Porous pressure-sensitive paint for characterizing unsteady flowfields," AIAA Journal 40, pp. 1094-1098, 2002.

6 Yorita, D., Nagai, H., Asai, K., Narumi, T., "Unsteady PSP technique for measuring naturallydisturbed periodic phenomena," 48th AIAA Aerospace Sciences Meeting Including the New Horizons Forum and Aerospace Exposition, Orlando, AIAA 2010-307, 2010. 
7 Asai, K., Yorita, D., "Unsteady PSP measurement in low-speed flow - overview of recent advancement at Tohoku University," 49th AIAA Aerospace Sciences Meeting Including the New Horizons Forum and Aerospace Exposition, Orlando, AIAA 2011-847, 2011.

${ }^{8}$ Liu, T., Teduka, N., Kameda, M., Asai, K., "Diffusion timescale of porous pressure-sensitive paint," AIAA Journal 39, pp. 2400-2402, 2001.

9 Kameda, M., Tabei, T., Nakakita, K., Sakaue, H., Asai, K., "Image measurements of unsteady pressure fluctuation by a pressure-sensitive coating on porous anodized aluminium," Measurement Science and Technology 16, pp. 2517-2524, 2005.

10 Sakaue, H., "Luminohpore application method of anodized aluminium pressure sensitive paint as a fast responding global pressure sensor," Review of Scientific Instruments 76, pp. 1-6, 2005.

11 Kameda, M., Tezuka, N., Hangai, T., Asai, K., Nakakita, K., Amao, Y., "Adsorptive pressuresensitive coatings on porous anodized aluminium," Measurement Science and Technology 15, pp. 489-500, 2004.

12 Gongora-Orozco, N., Zare-Behtash, H., Kontis, K., "Global unsteady pressure-sensitive paint measurements of a moving shock wave using thin-layer chromatography," Measurement 43, pp. $152-155,2010$.

13 Zare-Behtash, H., Gongora-Orozco, N., Kontis, K., Holder, S.J., "Application of novel pressuresensitive paint formulations for the surface flow mapping of high-speed jets," Experimental Thermal and Fluid Science 33, pp. 852-864, 2009.

14 Gongora-Orozco, N., Zare-Behtash, H., Kontis, K., "Effects of filters on the performance and characteristics of pressure-sensitive paints," Measurement Science and Technology 20, pp. 1-6, 2009 .

15 Schairer, E.T., "Optimum thickness of pressure-sensitive paint for unsteady measurements," AIAA Journal 40, pp. 2312-2318, 2002. 


\section{List of Figures}

1 SEM of anodized aluminium sample with no paint. . . . . . . . . . . . 9

2 SEM surface profiles for the (a) dipped and (b) sprayed sample. . . . . . . . 9

3 Surface intensity profiles: (a) dipped, (b) sprayed with 9 layers, (c) sprayed with 30 layers. . . . . . . . . . . . . . . . . . . . . . . . 9 9

4 SEM backscatter showing the surface texture of the sprayed AA sample. . . 10

5 Stern-Volmer plot for the different samples. . . . . . . . . . . . . . . . . . 10

6 Temperature sensitivity of AA-PSP samples. . . . . . . . . . . . . . . . . 11 


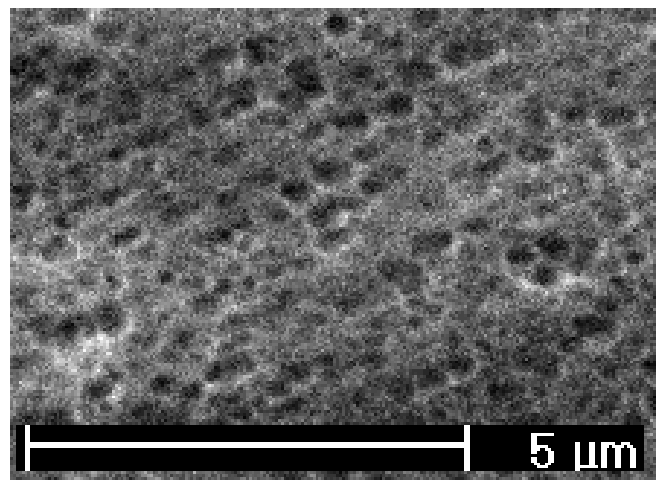

FIG. 1: SEM of anodized aluminium sample with no paint.

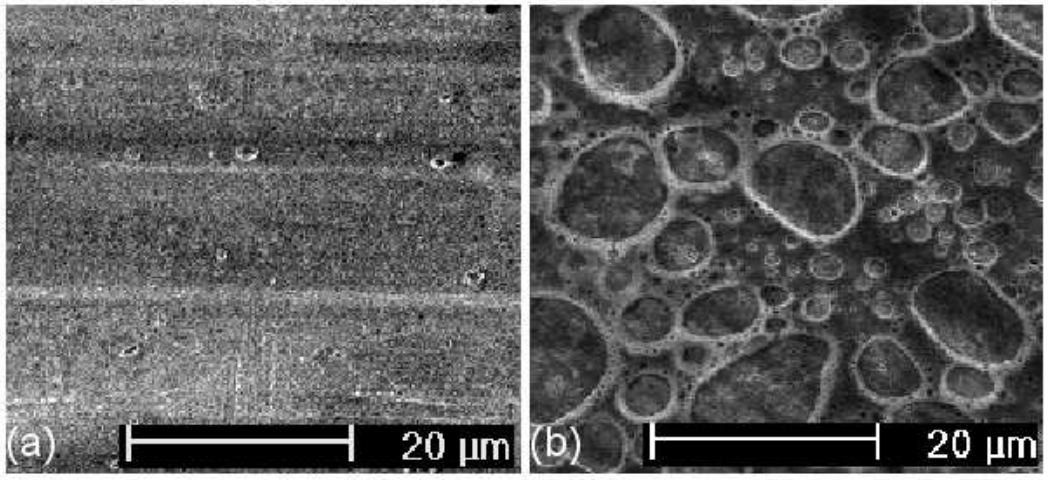

FIG. 2: SEM surface profiles for the (a) dipped and (b) sprayed sample.
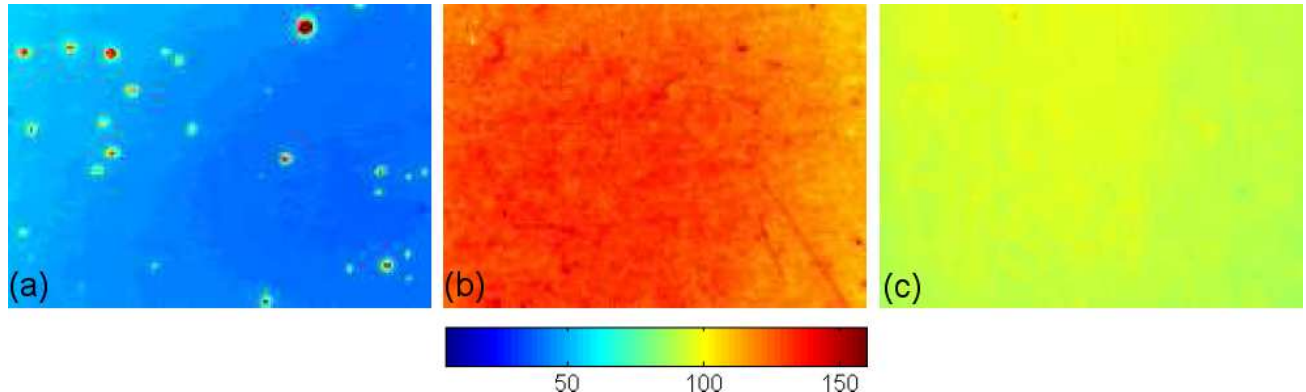

50

FIG. 3: Surface intensity profiles: (a) dipped, (b) sprayed with 9 layers, (c) sprayed with 30 layers. 


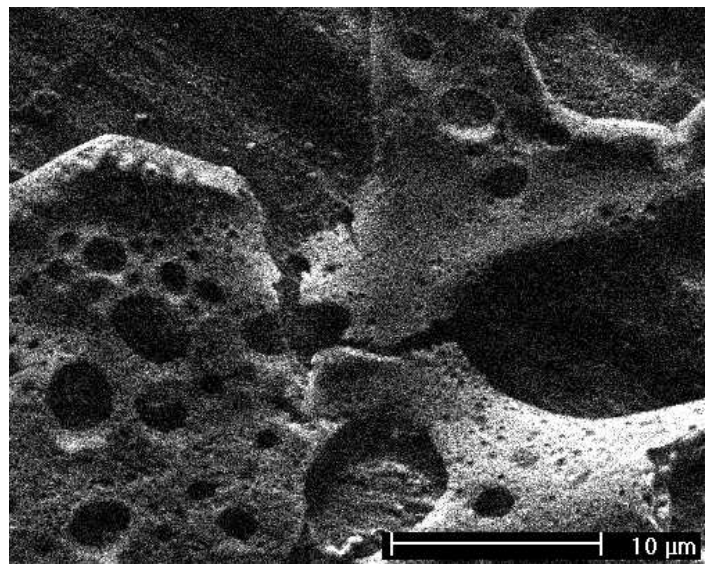

FIG. 4: SEM backscatter showing the surface texture of the sprayed AA sample.

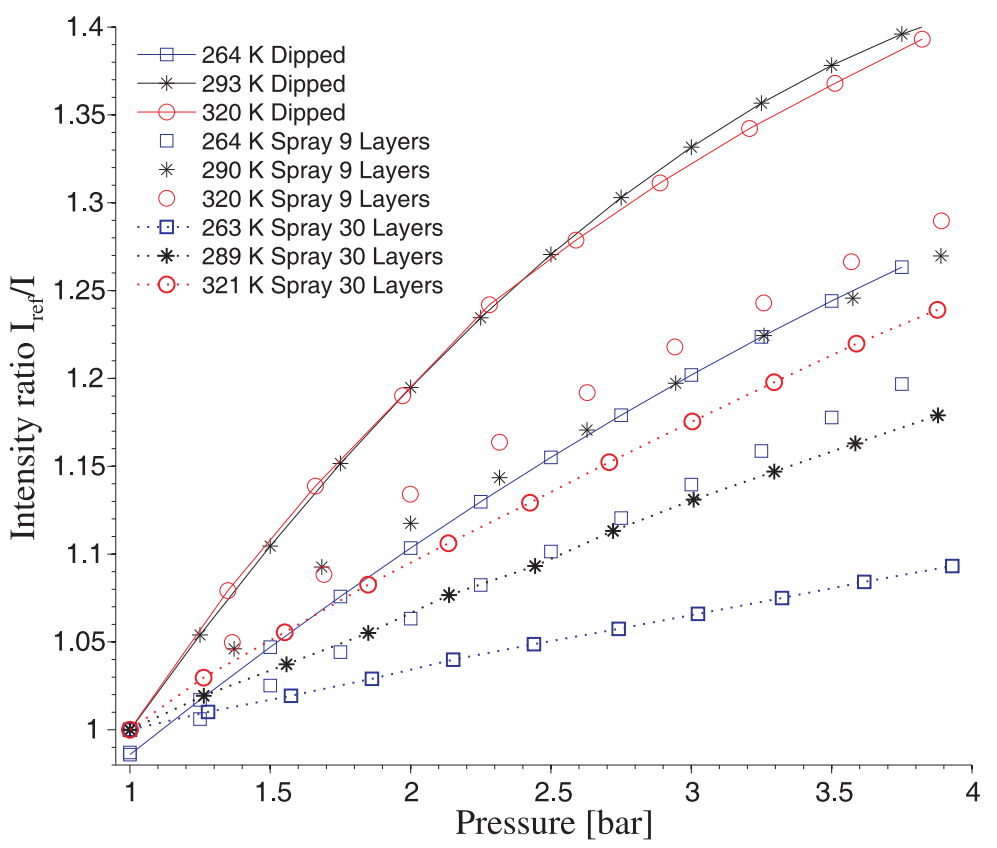

FIG. 5: Stern-Volmer plot for the different samples. 


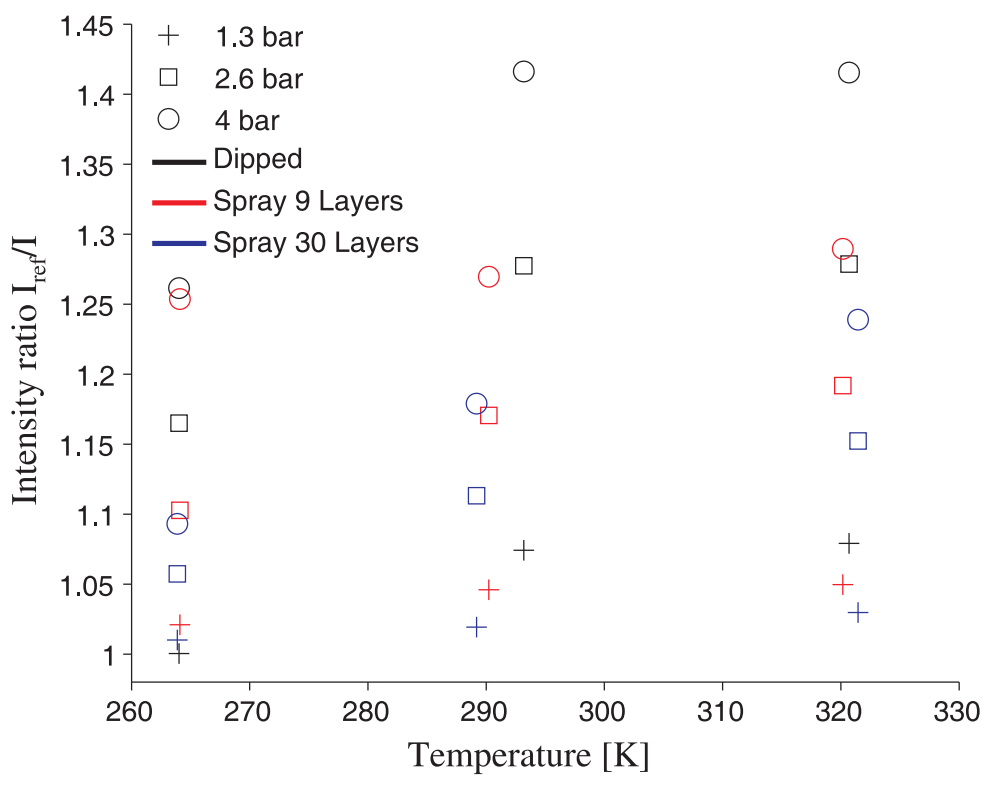

FIG. 6: Temperature sensitivity of AA-PSP samples. 